\title{
Association Between Hospital Rates of Early Do-Not-Resuscitate Orders and Favorable Neurological Survival Among Survivors of In-Hospital Cardiac Arrest
}

\author{
Timothy Fendler, MD ${ }^{1}$, John A. Spertus, MD, MPH ${ }^{1}$, Kevin Kennedy, MS ${ }^{1}$, Lena M. Chen, \\ MD, MS ${ }^{2}$, Sarah M. Perman, MD, MSCE ${ }^{3}$, and Paul S. Chan, MD, MSc ${ }^{1}$ for the American \\ Heart Association's Get With the Guidelines-Resuscitation Investigators \\ ${ }^{1}$ Department of Cardiology, Saint Luke's Mid America Heart Institute, Kansas City, MO \\ ${ }^{2}$ Department of Medicine, University of Michigan, Ann Arbor, MI \\ ${ }^{3}$ Department of Emergency Medicine, University of Colorado School of Medicine, Denver, CO
}

\section{Abstract}

Background-Current resuscitation guidelines recommend deferring accurate prognostication after in-hospital cardiac arrest until after 48-72 hours. Among patients who survive in-hospital cardiac arrest, it is unknown whether hospitals vary in making patients DNR early after a successful resuscitation and whether a hospital's rate of early DNR is associated with its rate of favorable neurological survival.

Methods-Within Get With the Guidelines-Resuscitation, we identified 24,899 patients from 236 hospitals who achieved return of spontaneous circulation (ROSC) after in-hospital cardiac arrest between 2006 and 2012. Hierarchical models were constructed to derive risk-adjusted hospital rates of DNR status adoption $\leq 2$ hours after ROSC and risk-standardized rates of favorable neurological survival (without severe disability; Cerebral Performance Category $\mathfrak{2}$ ). The association between a hospital's adjusted rate of early DNR and its rate of favorable neurological survival was evaluated using correlation statistics.

Results-Of 236 hospitals, $61.7 \%$ were academic, $74 \%$ had $\geq 200$ beds, and 94\% were urban. Overall, 5577 (22.4\%) patients were made DNR $\leq 12$ hours after ROSC, and risk-adjusted hospital rates of early DNR varied widely ( $7.1 \%$ to $40.5 \%$, median: $22.7 \%$ [IQR: $19.3 \%$ to $26.1 \%$ ]; median OR of 1.48). There was also significant hospital variation in risk-standardized rates for favorable neurological survival (3.5\% to $44.8 \%$, median: $25.3 \%$ [IQR: $20.2 \%$ to $29.4 \%$ ]; median OR 1.72 ). Notably, there was an inverse relationship between a hospital's risk-adjusted rate of early DNR and its risk-standardized rate of favorable neurological survival $(r=-0.179, \mathrm{p}=0.006)$.

Send correspondence and reprint requests to: Timothy Fendler, MD, Saint Luke's Mid-America Heart Institute, 4401 Wornall Rd., SLNI Suite \#5603, Kansas City, MO 64111, Phone: (816) 932-5475, Fax: (816) 932-5613, fendlert@umkc.edu.

DISCLOSURES

The content is solely the responsibility of the authors and does not necessarily represent the official views of the NIH.

Otherwise, all other coauthors have no relevant disclosures or potential conflicts of interest. 
Conclusions-Despite current guideline recommendations, many patients with in-hospital cardiac arrest are made DNR within 12 hours after ROSC, and hospitals vary widely in rates of early DNR. Higher hospital rates of early DNR were associated with worse survival outcomes.

\section{INTRODUCTION}

In-hospital cardiac arrest occurs in about 200,000 patients in the United States annually and is associated with a low survival rate of about $20 \%$ or less. ${ }^{1}$ Given this poor prognosis, discussions regarding resuscitation preferences are reasonable and appropriate for patients who survive an arrest event. ${ }^{2}$ However, a patient's decision to adopt Do-Not-Resuscitate (DNR) status only reflects the preference to have cardiopulmonary resuscitation withheld in the event of a recurrent cardiopulmonary arrest; it should not affect decisions about other medical treatments. Yet, previous studies have shown that DNR status adoption for other medical conditions is associated with lower rates of guideline-adherent treatment and higher mortality. ${ }^{3-5}$ This suboptimal care may be due to clinicians misinterpreting DNR preferences and thus not providing other appropriate therapeutic interventions (e.g., intensive care unit transfer or blood transfusion) $)^{6,7}$ or unwarranted pessimism about prognosis in patients made DNR thus leading to a "self-fulfilling prophecy" of poor outcomes. ${ }^{8}$

For patients with in-hospital cardiac arrest who have successful return of spontaneous circulation (ROSC), decisions about future resuscitative efforts are especially difficult in the early post-arrest period because a patient's prognosis may not become clear and evident for as long as 48 to 72 hours after cardiac arrest. ${ }^{9}$ Yet, it is likely that some hospitals are more aggressive in making patients DNR soon after achieving ROSC. Given wide hospital variation in rates of survival for patients with in-hospital cardiac arrest, ${ }^{10}$ it is unknown whether hospitals with higher rates of DNR adoption immediately after achieving ROSC have worse outcomes. If so, this may represent a potentially modifiable process, especially in sites with high rates of early DNR adoption.

Accordingly, we leveraged data from Get With the Guidelines (GWTG)-Resuscitation, a large national registry of in-hospital cardiac arrest, to assess whether high hospital rates of DNR in the early period after successful resuscitation from in-hospital cardiac arrest are associated with lower rates of favorable neurological survival (i.e., without severe cognitive disability). If this inverse association exists across hospitals, it may suggest the need for some hospitals to defer decisions about DNR status for a longer period after achieving ROSC (e.g., 2 to 3 days) and ensure that those made DNR are not routinely deprived of other aspects of appropriate care after being resuscitated.

\section{METHODS}

Study Design

Sponsored by the American Heart Association, GWTG-Resuscitation is a large, multicenter, observational registry of in-hospital cardiac arrests among U.S. hospitals that was begun in 2000. Hospital participation in the registry is voluntary, and details of the registry have been previously described in detail. ${ }^{11}$ In short, trained research personnel at each 
participating hospital identify and enroll all patients with in-hospital cardiac arrest (defined as unresponsiveness, apnea, and absence of a palpable central pulse), without prior DNR orders, and who have undergone cardiopulmonary resuscitation (CPR). This is accomplished through multiple sources of case identification, including medical records, centralized cardiac-arrest flow sheets, hospital paging-system logs, code cart checks, pharmacy tracer drug records, and hospital billing charges for use of resuscitation medications. ${ }^{11,12}$ Variables are collected prospectively and divided into 6 major categories: facility data, patient demographic data, pre-event data, event data, outcome data, and quality improvement data. ${ }^{11}$ Standardized data collection methods, including Utstein consensus definitions for all variables and outcomes, and strict oversight across all participating centers ensure accuracy, uniformity and completeness of the data. ${ }^{13-15}$ Outcome, A Quintiles Company, is the data collection coordination center for the American Heart Association/American Stroke Association Get With The Guidelines ${ }^{\circledR}$ programs

\section{Study Population}

Information on DNR status was introduced into the data collection form of GWTGResuscitation in April of 2006. Thus, our original cohort consisted of 72,875 patients who were 18 years or older and had a documented pulseless in-hospital cardiac arrest between April 2006 and September 2012 (Figure 1). For patients with more than one in-hospital cardiac arrest during a hospitalization, we excluded recurrent $(n=7,186)$ in-hospital cardiac arrests. For the purposes of this study, in which we assessed hospital rates of DNR status after successful resuscitation from in-hospital cardiac arrest, we excluded 31,224 patients who died during the acute resuscitation (i.e., did not achieve ROSC). We also excluded 2,179 patients from hospitals that did not routinely collect information on DNR status after a successful resuscitation. To focus on patients who arrested in either general inpatient or intensive care units (a more homogenous group with respect to causes and characteristics of cardiac arrests), we also excluded 7,311 patients who experienced in-hospital cardiac arrest in the emergency department, operating room, procedural and post-procedural areas. Additionally, we excluded patients with missing data on neurological status if alive at discharge (1,863 patients), as this variable comprised one of our study outcomes, and 3,971 patients for whom we could not calculate timing of DNR decisions due to missing or implausible times. There were no significant differences in baseline characteristics between these patients with missing data and the study cohort (Supplemental Table 1). Finally, we excluded 1,428 patients from hospitals with fewer than 20 patients to ensure each site had sufficient sample size for this study. Our final cohort comprised 24,899 patients who were successfully resuscitated after in-hospital cardiac arrest from 236 hospitals.

\section{Independent Variable and Study Outcome}

Our study examined the relationship between hospital rates of early DNR status adoption after successful resuscitation from in-hospital cardiac arrest and corresponding hospital rates of favorable neurological survival to discharge. Since many patients who eventually die become DNR closer to the time of death, and as we were interested in examining whether early DNR decisions correlated with worse survival outcomes, we defined DNR status as a patient for whom a DNR order was placed within 12 hours after achieving ROSC from an in-hospital cardiac arrest. Successfully resuscitated patients without DNR orders at any time 
during their admission or those with a DNR order placed more than 12 hours after successful resuscitation were defined as non-DNR. To further investigate the impact of using a threshold of 12 hours to define DNR status, we conducted a sensitivity analysis in which DNR status was instead defined as any patient for whom a DNR order was placed within 72 hours after achieving ROSC from an in-hospital cardiac arrest.

The primary outcome, favorable neurological survival, was defined as survival to hospital discharge without severe neurological disability. Neurological disability in GWTGResuscitation is measured by Cerebral Performance Category (CPC) scores, wherein a CPC of 1 is assigned to patients at discharge with little to no neurological disability, 2 with moderate disability, 3 with severe disability, and 4 for those in a persistent coma or vegetative state. Based on prior work, favorable neurological survival was defined as alive at hospital discharge with a CPC score of 1 or $2,{ }^{16}$ referring to patients with no deficits or those who can still perform independent activities of daily living. ${ }^{17}$ We also conducted a secondary analysis in which the outcome was survival to hospital discharge, regardless of neurological status at discharge.

\section{Statistical Analysis}

Hospital Rate of Early DNR-We used summary statistics to describe the baseline characteristics of hospitals included in our analysis and simple proportions to calculate each hospital's rate of early DNR among survivors of in-hospital cardiac arrest. Since hospitals may vary in patient case-mix, we constructed a hierarchical, multivariable logistic regression model, with site as a random effect, to compute risk-adjusted rates of early DNR status adoption among hospitals. This model adjusted for patients' age, sex, race, pre-arrest comorbidities (history of myocardial infarction, congestive heart failure, stroke, diabetes, pneumonia, respiratory insufficiency, hepatic insufficiency, renal insufficiency, malignancy, and atrial arrhythmia, as well as myocardial infarction, heart failure, hypotension, trauma, and septicemia during the index hospitalization), and arrest characteristics, including presenting rhythm, event location, duration of acute resuscitation, various pre-arrest interventions in place, and time and day of arrest.

To characterize patients across sites, we then divided hospitals into tertiles by their adjusted rate of early DNR and compared hospital rates of patient characteristics across tertiles using Kruskal-Wallis tests for continuous variables and Mantel-Haenszel trend tests for categorical variables. To quantify hospital variation in rates of early DNR after in-hospital cardiac arrest, we calculated median odds ratios (OR), which assess the likelihood that patients with identical patient-level covariates would be made DNR early after ROSC at one randomly chosen hospital compared with another. The median OR is derived from our aforementioned hierarchical model with only patient-level factors included. A median OR of 1 suggests no variation across hospitals, whereas a median OR of 1.50 suggests a patient has a $50 \%$ difference in odds of being made DNR between two randomly selected hospitals. ${ }^{18-20}$

Association Between Hospital DNR rates and Outcomes-We first calculated riskstandardized survival outcomes for each hospital. Using the validated Cardiac Arrest Survival Post-Resuscitation In-hospital (CASPRI) model, which has been shown to strongly 
predict one's likelihood of favorable neurological survival (c-statistic of 0.802) and have excellent calibration, ${ }^{16}$ we calculated risk-standardized rates of favorable neurological survival for each hospital. We then evaluated the association between a hospital's riskadjusted rate of early DNR and its risk-standardized rate of favorable neurological survival using scatter plots and by computing a Pearson's correlation coefficient between these 2 risk-adjusted rates.

The Institutional Review Board (IRB) of the Mid-America Heart Institute approved this study and waived the requirement for informed consent. For all analyses, the null hypothesis was evaluated at a two-side significance level of 0.05 with $95 \%$ confidence intervals (CIs). All analyses were conducted with SAS 9.1 (SAS Institute, Cary, NC) and R version 2.6.2.21

\section{RESULTS}

Of 236 hospitals in the cohort, $61.7 \%$ were academic hospitals, $83.0 \%$ had $\geq 200$ beds, and $94 \%$ were urban centers (Table 1). There were a total of 24,899 patients with in hospital cardiac arrest in these hospitals. The mean age was $65.0 \pm 15.7$ years, $43.5 \%$ were female and $69.3 \%$ were white (Supplemental Table 2). After achieving ROSC, 5577 (22.4\%) patients were made DNR within the first 12 hours, and a total of 6039 (24.3\%) patients survived to discharge with a favorable neurological outcome.

\section{Variation in Hospital Rates of Early DNR Status Adoption}

Unadjusted rates of early DNR status adoption varied across hospitals, with a range of $0 \%$ to $58.3 \%$ and a median rate of $23.2 \%$ (inter-quartile range: $17.3 \%$ to $28.6 \%$; Figure 2). After adjustment for demographics, comorbidities, and event characteristics, the range of early DNR status adoption was $7.1 \%$ to $40.5 \%$, and the median rate remained similar at $22.7 \%$ (inter-quartile range: $19.3 \%$ to $26.1 \%$ ). The adjusted median OR for early DNR adoption was 1.48 , suggesting a $48 \%$ increased odds of being made DNR early after ROSC at one random hospital compared with another in a patient with similar baseline and cardiac arrest characteristics.

Hospitals in the lowest tertile of early DNR rates made $7.2 \%$ to $20.4 \%$ of their in-hospital cardiac arrest survivors DNR within the first 12 hours, whereas hospitals in the highest tertile had early DNR rates of $24.9 \%$ to $40.5 \%$ (Table 2). In general, hospitals in each tertile had similar rates of comorbid conditions, and there were no differences in cardiac arrest rhythm, location of cardiac arrest (e.g., ICU, unmonitored floor), and whether arrests occurred during daytime vs. nighttime by hospital rates of early DNR. Hospitals in the tertile with the highest rate of early DNR did have patients who were older and had higher rates of hypotension or coexisting pneumonia at the time of cardiac arrest. In contrast, hospitals in the lowest tertile had a higher proportion of patients on intravenous vasopressors at the time of cardiac arrest and their patients required longer resuscitations prior to achieving ROSC.

\section{Association of Early DNR Status Adoption with Favorable Neurological Survival}

Risk-standardized hospital rates of favorable neurological survival varied across hospitals, with a range of $3.5 \%$ to $44.8 \%$ and a median rate of $25.3 \%$ (inter-quartile range of $20.2 \%$ to $29.4 \%$ ). The adjusted median OR for favorable neurological survival was 1.72, suggesting 
substantial variation in survival outcomes across sites. Notably, hospital rates of early DNR for in-hospital cardiac arrest were strongly and inversely correlated with risk-standardized rates of favorable neurological survival ( $r$ of $-0.179, p=0.006$; Figure 3 ), which suggests that hospitals which were most aggressive at making patients DNR early after ROSC had the lowest risk-standardized rate of favorable neurological outcome, and vice versa. Similar inverse correlations were found in sensitivity analyses defining early DNR as within 72 hours of ROSC ( $\mathrm{r}$ of $-0.114, \mathrm{p}=0.079$ ) and defining survival as any survival to discharge ( $\mathrm{r}$ of $-0.137, \mathrm{p}=0.031)$.

\section{DISCUSSION}

In this large, national registry, we found that there was wide variation in hospital rates of early DNR status adoption among patients who survived an in-hospital cardiac arrest event, even as most patient and cardiac arrest characteristics were similar across hospitals. Indeed, the median OR for early DNR adoption by successfully resuscitated patients was 1.48 across sites, implying an almost 50\% variability in the odds of being made DNR between two hospitals despite similar patient characteristics. Hospitals which made a greater proportion of their successfully resuscitated patients DNR within the first 12 hours after ROSC had lower rates of favorable neurological survival, even after adjusting for patient characteristics, comorbid diseases, severity of illness, and cardiac arrest details (including code duration). Collectively, these results suggest that decisions for making patients DNR immediately after ROSC are not consistent across hospitals and this variability may also be linked to some of the observed hospital variation in survival outcomes after in-hospital cardiac arrest.

For other clinical conditions, point estimates of DNR adoption rates have been reported to vary widely, from $9 \%$ to $35 \%^{3,22-26}$. In such studies, patient-level DNR status was associated with higher mortality. ${ }^{5,26-28}$ Moreover, patients made DNR were less likely to receive appropriate medical care unrelated to emergent resuscitation, such as intensive care unit transfer, intravenous antihypertensive therapy, and deep venous thrombosis prophylaxis for intracranial hemorrhage, ${ }^{3}$ cardiac catheterization and internal cardiac defibrillator placement for out-of-hospital cardiac arrest, ${ }^{25}$ and left ventricular function assessment, renin-angiotensin system blockade, and diet/lifestyle counseling for heart failure. ${ }^{4}$ Finally, four studies have previously addressed variation across hospitals in regard to early DNR status adoption and associated survival outcomes, albeit in different cohorts of patients with intracranial hemorrhage, ${ }^{22}$ ischemic stroke, ${ }^{24}$ traumatic brain injury, ${ }^{29}$ or out-of-hospital cardiac arrest ${ }^{25}$ (a very different population than that of patients with in-hospital arrest events ${ }^{30,31}$ ). As with our study results, all four prior studies also reported wide variation in hospital rates of DNR status adoption, from 0 to $80 \%$, and worse survival associated with higher hospital rates of DNR.

We extended the findings of prior investigations by examining hospital variation in DNR rates for in-hospital cardiac arrest and its association with favorable neurological survival, a more meaningful outcome for patients than any survival, regardless of quality. In-hospital cardiac arrest is an ideal condition for examining this relationship as it is associated with a low survival rate and the timing of decisions for DNR status is easy to quantify. Although there may be compelling reasons to make a patient DNR, such as severity of illness, overall 
prognosis, and patient or family preferences, we found that sites varied widely in how aggressively they established DNR status within 12 hours of the acute resuscitation despite treating very similar patients with in-hospital cardiac arrest. In turn, sites that were less aggressive in making patients DNR altogether or that waited longer to establish DNR in patients were associated with higher overall rates of favorable neurological outcome.

Current resuscitation guidelines recommend waiting 48-72 hours after successful resuscitation from in-hospital cardiac arrest to better assess a patient's prognostic trajectory to allow for more accurate neurological prognosis, as this may not become evident in the immediate aftermath of resuscitation from cardiac arrest. ${ }^{9}$ While prior studies have used a definition of early DNR that includes the first 24 hours after admission, we defined early DNR as within 12 hours of a discrete in-hospital event to which the decision might be more reliably related, in order to highlight that these earlier decisions for resuscitation code status are being made before the time frame recommended for prognostication. Although most patient and cardiac arrest characteristics were similar across hospitals, we did find that two of the strongest prognostic factors for favorable neurological survival-resuscitation time and requirement for continuous intravenous vasopressors at the time of cardiac arrest-were more prevalent in the hospital tertile with the lowest early DNR rate, suggesting that unmeasured patient severity of illness is unlikely to fully account for the inverse association between a hospital's early DNR rate and its rate of favorable neurological outcome. In fact, it is quite possible that hospitals with higher rates of early DNR are also less aggressive in other aspects of intensive medical care for its acute survivors of in-hospital cardiac arrest, which may explain their worse survival outcomes. This deserves further study, as a hospital's early DNR rate and its treatment of patients once made DNR - both modifiable processes-may represent potential opportunities to improve survival outcomes for inhospital cardiac arrest, given wide variation in survival outcomes across hospitals. Such efforts would also be patient-centered, as they would ensure that successfully resuscitated patients are given adequate time for accurate neurological prognostication and remain aggressively treated (outside of a recurrent cardiopulmonary arrest) to optimize outcomes.

Our results should be taken in the context of a number of limitations. Despite our finding of an association between a hospital's rate of early DNR status adoption and worse survival outcome, it remains unknown whether early DNR is a marker or mediator of survival. We did not have information from GWTG-R to determine what prognostic criteria clinicians used to discuss resuscitation code status with patients. Moreover, there may be unmeasured confounders that would account for differences in hospital DNR rates and survival outcomes (e.g., frailty). Nonetheless, it is reassuring that some of the strongest predictors of a nonfavorable neurological outcome (resuscitation duration and vasopressors) were more prevalent in hospitals with the lowest early DNR rate. Second, we did not have detailed information as to whether other aspects of care differed among DNR and non-DNR patients across sites to inform whether DNR patients received less aggressive post-resuscitation care. Finally, GWTG-R does not collect detailed information on whether discussions regarding DNR status occurred between patients, their families and the health care team, what their contents were, and what preferences patients or their families might espouse (e.g., spiritual or religious beliefs) that could influence their willingness to receive aggressive post- 
resuscitation care. Such unmeasured variables could further mediate the association between hospital rates of DNR status adoption and favorable neurological survival.

In conclusion, we found marked variability across U.S. hospitals in making patients DNR within 12 hours after successful resuscitation from an in-hospital cardiac arrest, despite current guidelines that recommend deferring more accurate prognostication until 48 to 72 hours after successful resuscitation. Hospitals that make a higher proportion of their patients DNR within 12 hours after in-hospital cardiac arrest, in turn, are associated with lower rates of favorable neurological survival. These disparities in care may be improved by better adherence to guideline recommendations to avoid assigning prognosis until 2 to 3 days after experiencing cardiac arrest, and by ensuring that patients who choose DNR status do not have aspects of care withheld inappropriately, but instead receive optimal treatment in accordance with their preferences.

\section{Supplementary Material}

Refer to Web version on PubMed Central for supplementary material.

\section{Acknowledgments}

FUNDING

Dr. Fendler is supported by a T32 grant from the NHLBI (T32HL110837).

Dr. Chan is funded by grants from the NHLBI (1R01HL123980 and K23HL102224).

\section{References}

1. Merchant RM, Yang L, Becker LB, et al. Incidence of treated cardiac arrest in hospitalized patients in the United States. Crit Care Med. Nov; 2011 39(11):2401-2406. [PubMed: 21705896]

2. Girotra S, Nallamothu BK, Spertus JA, Li Y, Krumholz HM, Chan PS. Trends in survival after inhospital cardiac arrest. N Engl J Med. Nov 15; 2012 367(20):1912-1920. [PubMed: 23150959]

3. Silvennoinen K, Meretoja A, Strbian D, Putaala J, Kaste M, Tatlisumak T. Do-not-resuscitate (DNR) orders in patients with intracerebral hemorrhage. Int J Stroke. Jan; 2014 9(1):53-58. [PubMed: 24148872]

4. Chen JL, Sosnov J, Lessard D, Goldberg RJ. Impact of do-not-resuscitation orders on quality of care performance measures in patients hospitalized with acute heart failure. Am Heart J. Jul; 2008 156(1):78-84. [PubMed: 18585500]

5. Shepardson LB, Youngner SJ, Speroff T, Rosenthal GE. Increased risk of death in patients with donot-resuscitate orders. Med Care. Aug; 1999 37(8):727-737. [PubMed: 10448716]

6. Krumholz HM, Phillips RS, Hamel MB, et al. Resuscitation preferences among patients with severe congestive heart failure: results from the SUPPORT project. Study to Understand Prognoses and Preferences for Outcomes and Risks of Treatments. Circulation. Aug 18; 1998 98(7):648-655. [PubMed: 9715857]

7. Beach MC, Morrison RS. The effect of do-not-resuscitate orders on physician decision-making. J Am Geriatr Soc. Dec; 2002 50(12):2057-2061. [PubMed: 12473020]

8. Becker KJ, Baxter AB, Cohen WA, et al. Withdrawal of support in intracerebral hemorrhage may lead to self-fulfilling prophecies. Neurology. Mar 27; 2001 56(6):766-772. [PubMed: 11274312]

9. Field JM, Hazinski MF, Sayre MR, et al. Part 1: executive summary: 2010 American Heart Association Guidelines for Cardiopulmonary Resuscitation and Emergency Cardiovascular Care. Circulation. Nov 2; 2010 122(18 Suppl 3):S640-656. [PubMed: 20956217] 
10. Merchant RM, Berg RA, Yang L, Becker LB, Groeneveld PW, Chan PS. Hospital variation in survival after in-hospital cardiac arrest. J Am Heart Assoc. 2014; 3(1):e000400. [PubMed: 24487717]

11. Peberdy MA, Kaye W, Ornato JP, et al. Cardiopulmonary resuscitation of adults in the hospital: a report of 14720 cardiac arrests from the National Registry of Cardiopulmonary Resuscitation. Resuscitation. Sep; 2003 58(3):297-308. [PubMed: 12969608]

12. Chan PS, Krumholz HM, Nichol G, Nallamothu BK. Delayed time to defibrillation after inhospital cardiac arrest. N Engl J Med. Jan 3; 2008 358(1):9-17. [PubMed: 18172170]

13. Cummins RO, Chamberlain D, Hazinski MF, et al. Recommended guidelines for reviewing, reporting, and conducting research on in-hospital resuscitation: the in-hospital 'Utstein style'. American Heart Association. Circulation. Apr 15; 1997 95(8):2213-2239. [PubMed: 9133537]

14. Jacobs I, Nadkarni V, Bahr J, et al. Cardiac arrest and cardiopulmonary resuscitation outcome reports: update and simplification of the Utstein templates for resuscitation registries: a statement for healthcare professionals from a task force of the International Liaison Committee on Resuscitation (American Heart Association, European Resuscitation Council, Australian Resuscitation Council, New Zealand Resuscitation Council, Heart and Stroke Foundation of Canada, InterAmerican Heart Foundation, Resuscitation Councils of Southern Africa). Circulation. Nov 23; 2004 110(21):3385-3397. [PubMed: 15557386]

15. Peberdy MA, Ornato JP, Larkin GL, et al. Survival from in-hospital cardiac arrest during nights and weekends. JAMA. Feb 20; 2008 299(7):785-792. [PubMed: 18285590]

16. Chan PS, Spertus JA, Krumholz HM, et al. A validated prediction tool for initial survivors of inhospital cardiac arrest. Arch Intern Med. Jun 25; 2012 172(12):947-953. [PubMed: 22641228]

17. Brain Resuscitation Clinical Trial I Study Group. A randomized clinical study of cardiopulmonarycerebral resuscitation: design, methods, and patient characteristics. Am J Emerg Med. Jan; 1986 4(1):72-86. [PubMed: 2868736]

18. Chan PS, Nichol G, Krumholz HM, Spertus JA, Nallamothu BK. Hospital variation in time to defibrillation after in-hospital cardiac arrest. Arch Intern Med. Jul 27; 2009 169(14):1265-1273. [PubMed: 19636027]

19. HG. Multilevel Statistical Models. London, England: Edward Arnold; 1995.

20. Larsen K, Petersen JH, Budtz-Jorgensen E, Endahl L. Interpreting parameters in the logistic regression model with random effects. Biometrics. Sep; 2000 56(3):909-914. [PubMed: 10985236]

21. R Development Core Team. R: A language and environment for statistical computing. Vienna, Austria: R Foundation for Statistical Computing; 2014. http://www.R-project.org/

22. Hemphill JC 3rd, Newman J, Zhao S, Johnston SC. Hospital usage of early do-not-resuscitate orders and outcome after intracerebral hemorrhage. Stroke. May; 2004 35(5):1130-1134. [PubMed: 15044768]

23. Jackson EA, Yarzebski JL, Goldberg RJ, et al. Do-not-resuscitate orders in patients hospitalized with acute myocardial infarction: the Worcester Heart Attack Study. Arch Intern Med. Apr 12; 2004 164(7):776-783. [PubMed: 15078648]

24. Kelly AG, Zahuranec DB, Holloway RG, Morgenstern LB, Burke JF. Variation in do-notresuscitate orders for patients with ischemic stroke: implications for national hospital comparisons. Stroke. Mar; 2014 45(3):822-827. [PubMed: 24523035]

25. Richardson DK, Zive D, Daya M, Newgard CD. The impact of early do not resuscitate (DNR) orders on patient care and outcomes following resuscitation from out of hospital cardiac arrest. Resuscitation. Apr; 2013 84(4):483-487. [PubMed: 22940596]

26. Zahuranec DB, Brown DL, Lisabeth LD, et al. Early care limitations independently predict mortality after intracerebral hemorrhage. Neurology. May 15; 2007 68(20):1651-1657. [PubMed: 17502545]

27. Chen YY, Connors AF Jr, Garland A. Effect of decisions to withhold life support on prolonged survival. Chest. Jun; 2008 133(6):1312-1318. [PubMed: 18198259]

28. Wenger NS, Pearson ML, Desmond KA, Brook RH, Kahn KL. Outcomes of patients with do-notresuscitate orders. Toward an understanding of what do-not-resuscitate orders mean and how they affect patients. Arch Intern Med. Oct 23; 1995 155(19):2063-2068. [PubMed: 7575065] 
29. Dean D, Martinez MS, Newgard CD. Variability in early do not attempt resuscitation orders among patients with serious traumatic brain injury. Acad Emerg Med. Jan; 2015 22(1):54-60. [PubMed: 25545694]

30. Fredriksson M, Aune S, Bang A, et al. Cardiac arrest outside and inside hospital in a community: mechanisms behind the differences in outcome and outcome in relation to time of arrest. Am Heart J. May; 2010 159(5):749-756. [PubMed: 20435182]

31. Herlitz J, Bang A, Ekstrom L, et al. A comparison between patients suffering in-hospital and outof-hospital cardiac arrest in terms of treatment and outcome. J Intern Med. Jul; 2000 248(1):5360. [PubMed: 10947881] 


\section{2,875 patients with index pulseless} cardiac arrests from 459 hospitals

\section{1,224 patients without ROSC}

2,179 patients at hospitals not routinely collecting DNR data

7,311 patients arrested outside general inpatient or intensive care units (ED, OR, et.)

3,971 patients with missing/implausible DNR times

1,863 palients alive at discharge without CPC scores

1,428 palients at hospitals with $<20$ palients eligible for study

\section{4,899 patients with complete} data from 236 hospitals

Figure 1. Patient Cohort Exclusion Flow Chart

ROSC, return of spontaneous circulation; DNR, do not resuscitate; ED, emergency department; OR, operating room; CPC, Cerebral Performance Category 

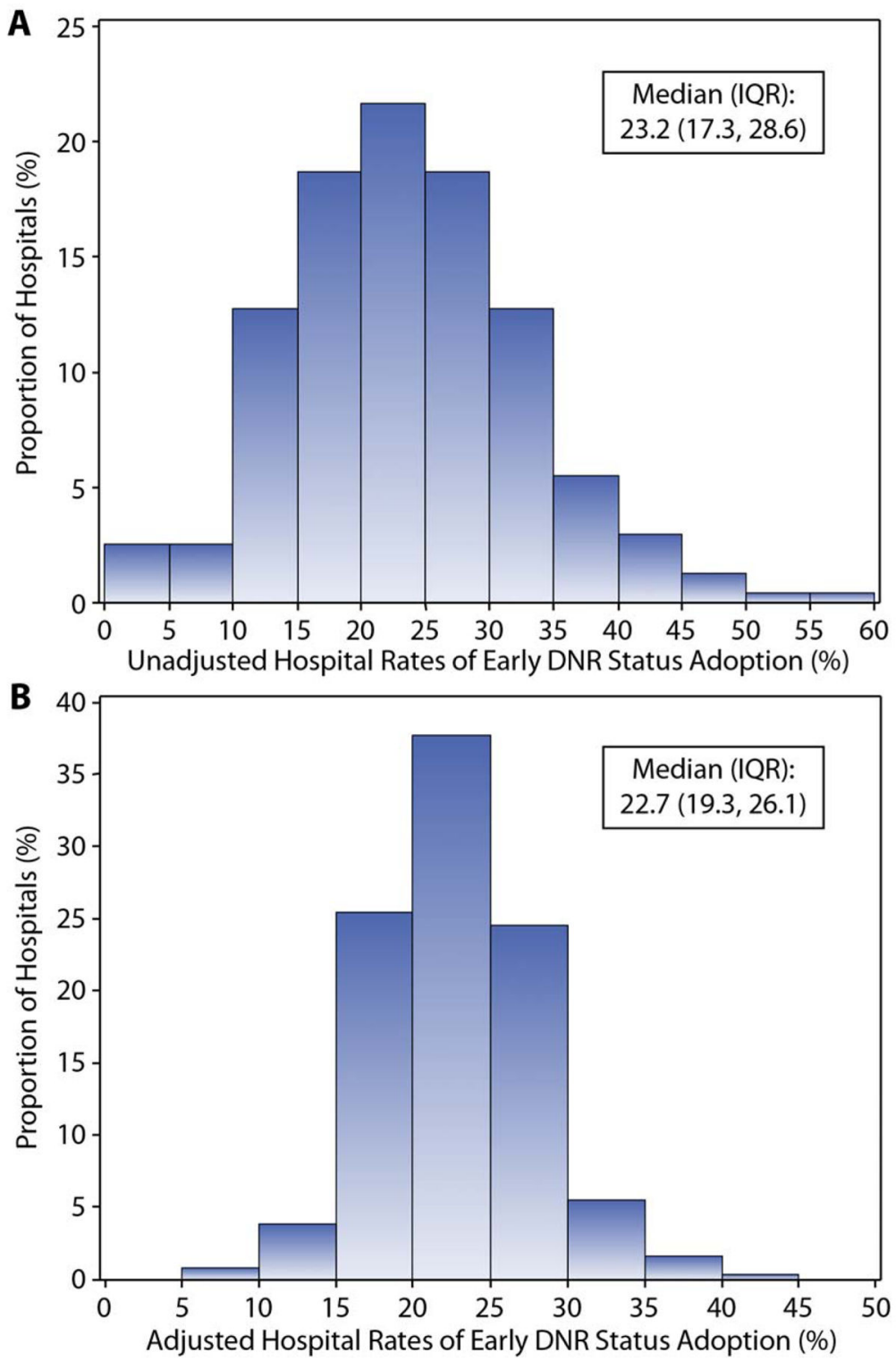

Figure 2. Distribution of Early DNR Status Adoption Rates, Across Hospitals

Hospital rates for early DNR status adoption, both, (A) unadjusted, and (B) after multivariable adjustment for demographics, comorbidities, and event characteristics. (DNR, do not resuscitate; IQR, interquartile range) 

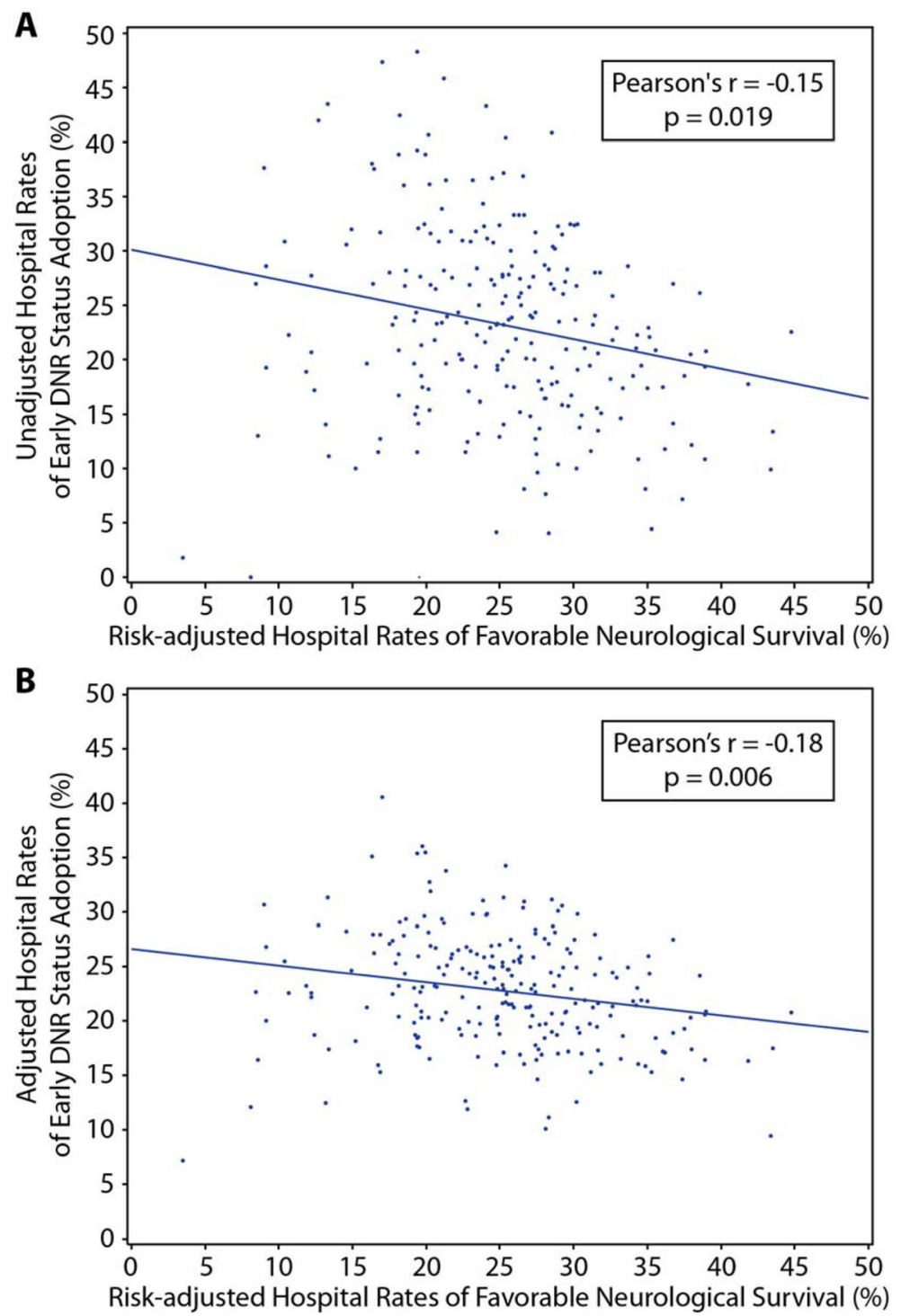

Figure 3. Association of Early DNR Status Adoption with Favorable Neurological Survival, Across Hospitals

Correlation between hospital rates of favorable neurological survival (adjusted for CASPRI score) and hospital rates of early DNR status adoption, both (A) unadjusted, and (B) after multivariable adjustment for demographics, comorbidities, and event characteristics. (DNR, do not resuscitate; CASPRI, Cardiac Arrest Survival Post-Resuscitation In-hospital) 


\section{Table 1}

\section{Baseline Characteristics of Participating Hospitals}

\begin{tabular}{|c|c|}
\hline Characteristics & Proportion $(\%)(n=236)$ \\
\hline \multicolumn{2}{|l|}{ Teaching Status } \\
\hline Academic & 61.7 \\
\hline Non-teaching & 38.2 \\
\hline \multicolumn{2}{|l|}{ Bed Size } \\
\hline Small (0-199) & 17.0 \\
\hline Medium (200-499) & 55.4 \\
\hline Large ( 2500$)$ & 27.6 \\
\hline \multicolumn{2}{|l|}{ Ownership } \\
\hline Non-profit & 52.5 \\
\hline Church-affiliated & 15.7 \\
\hline State/Local Government & 14.7 \\
\hline Private/Investors & 13.8 \\
\hline VA/Military & 3.2 \\
\hline \multicolumn{2}{|l|}{ Region } \\
\hline North Mid-Atlantic & 13.8 \\
\hline South Atlantic & 27.6 \\
\hline North Central & 21.7 \\
\hline South Central & 19.4 \\
\hline Mountain/Pacific & 17.5 \\
\hline \multicolumn{2}{|l|}{ Location } \\
\hline Rural & 6.0 \\
\hline Urban & 94.0 \\
\hline \multicolumn{2}{|l|}{ Trauma Center Level } \\
\hline 1 (Regional) & 38.8 \\
\hline 2 (Community) & 41.0 \\
\hline 3 (Rural) & 19.4 \\
\hline 4 (State-defined) & 0.7 \\
\hline Urgent Care Center & 42.9 \\
\hline
\end{tabular}

Am Heart J. Author manuscript; available in PMC 2018 October 01. 
Fendler et al.

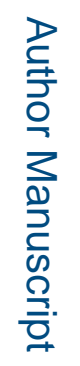

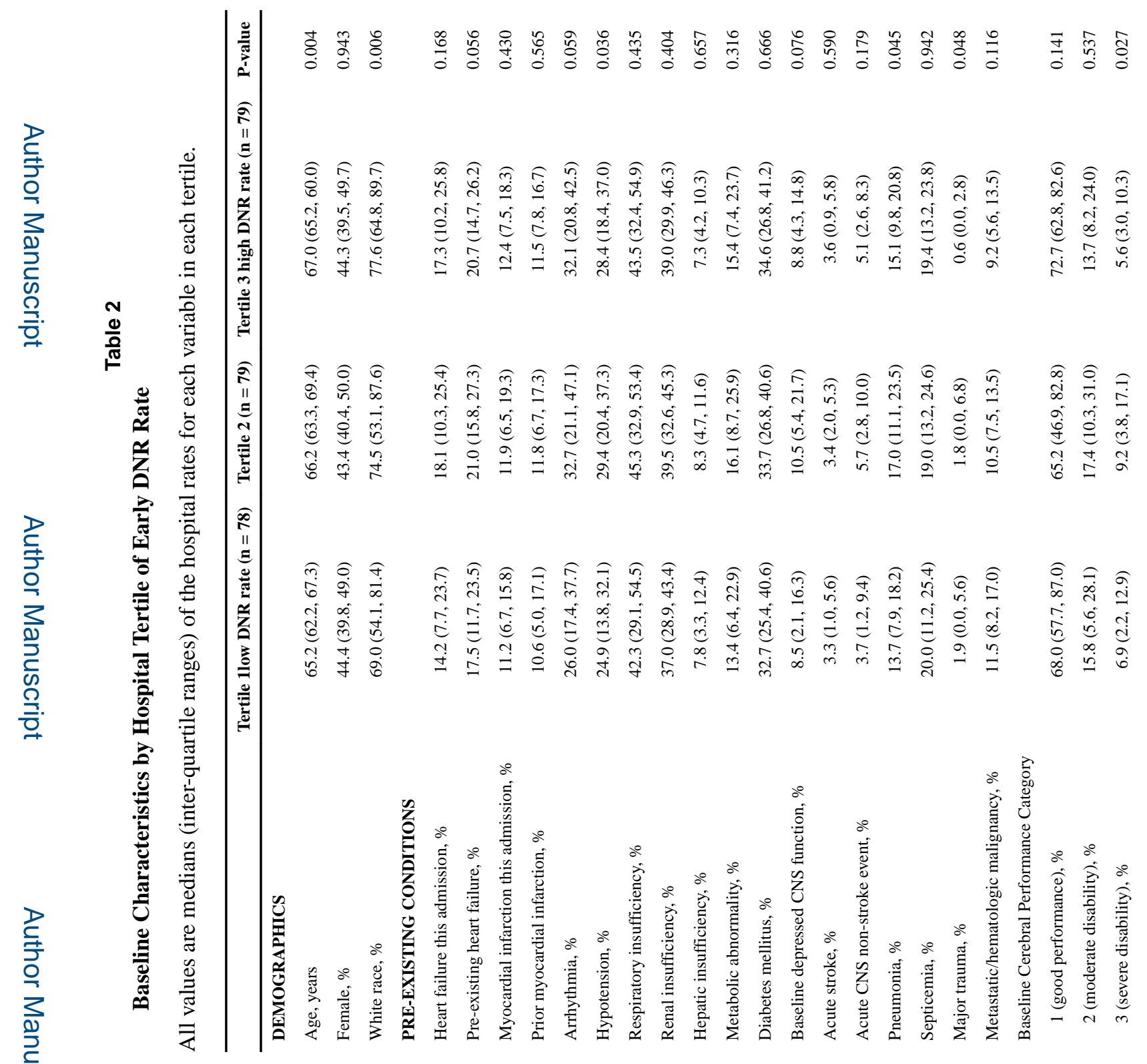

Am Heart J. Author manuscript; available in PMC 2018 October 01. 
Fendler et al.

Page 16

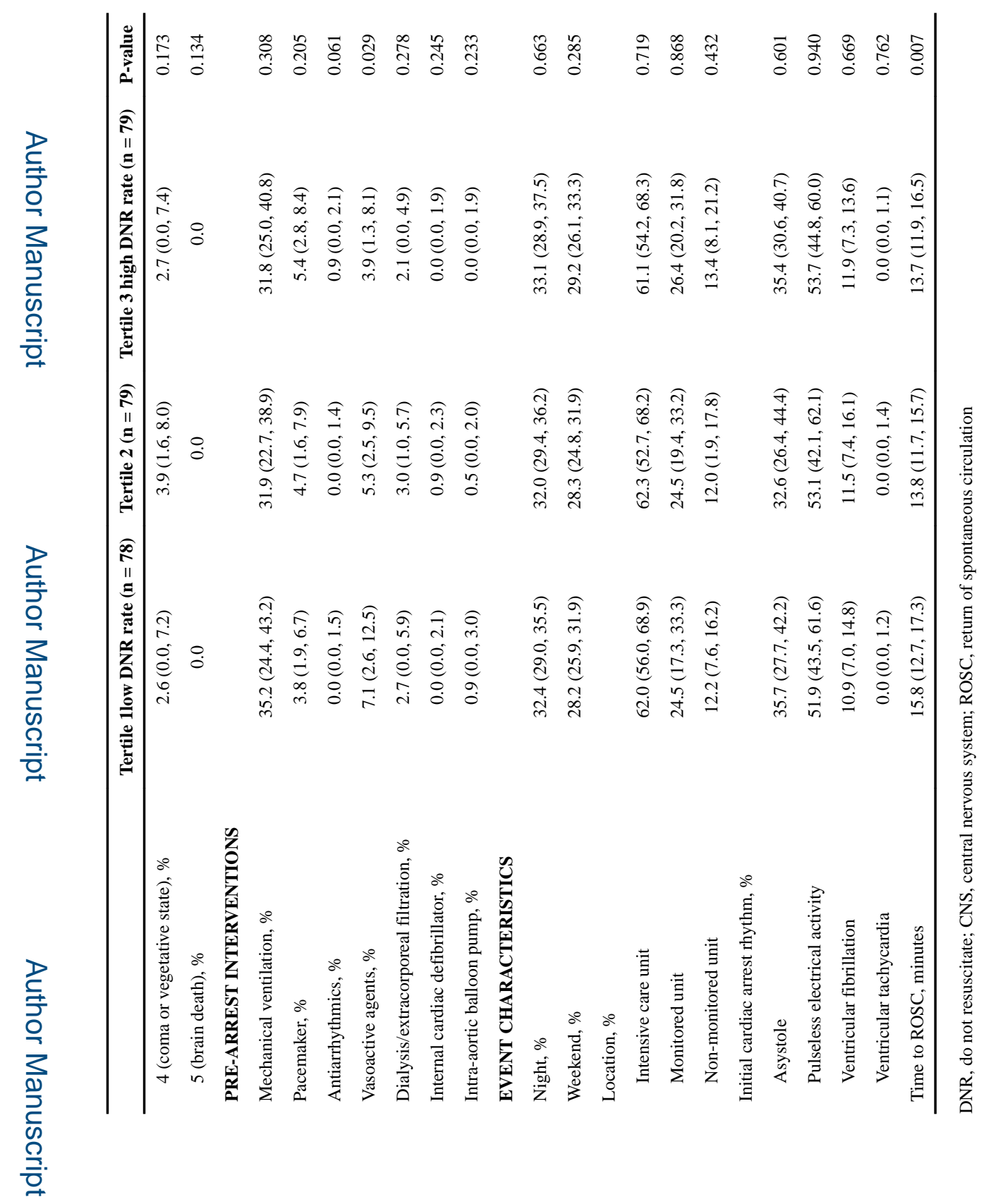

로을

Am Heart J. Author manuscript; available in PMC 2018 October 01. 